\title{
Clinicopathologic Features and Treatment Characteristics of Congenital Corneal Opacity Infants and Children Aged 3 Years or Less: A Retrospective Single Institution Analysis
}

\author{
Sen Miao a,b Qi Lin ${ }^{c}$ Yang Liu ${ }^{d}$ Yao-Wen Song ${ }^{a}$ Ying-Nan Zhang ${ }^{a}$ \\ Zhi-Qiang Pan ${ }^{a}$
}

aBeijing Ophthalmology and Visual Science Key Laboratory, Beijing Tongren Eye Center, Beijing Tongren Hospital, Capital Medical University, Beijing, China; ${ }^{b}$ Department of Ophthalmology, Beijing Anzhen Hospital, Beijing, China; 'Department of Ophthalmology, Beijing Children's Hospital, Capital Medical University, National Center for Children's Health, Beijing, China; ${ }^{d}$ Department of Ophthalmology, The First Hospital of Lanzhou University, Chengguan District, Lanzhou, China

\section{Highlights}

- Clinicopathologic features of 90 infants and children with CCO were studied.

- CCO has varied manifestations in infants and children in China.

- A thorough medical history and careful clinical examination are critical for accurate diagnosis and classification of CCO.

\section{Keywords}

Congenital corneal opacity · Infants - Clinicopathologic features · Diagnosis

\begin{abstract}
Objective: In this retrospective single institution study, we investigated the clinicopathologic features and treatment characteristics of 90 patients with congenital corneal opacities (CCO) (117 eyes) who were 3 years and younger and treated at our hospital. Subject and Methods: We reviewed the clinical data of patients with CCO who presented for the first time for treatment at our hospital between January 1, 2017, and December 31, 2017. CCO were classified using the "STUMPED" (Sclerocornea, Tears in Descement's membrane, Metabolic, Peters, Endothelial dystrophy and Dermoid) method and confirmed by pathological examination.
\end{abstract}

\begin{tabular}{ll}
\hline KARGER & $\begin{array}{l}\text { ( 2019 The Author(s) Karger } \\
\text { Published by S. Karger AG, Basel }\end{array}$ \\
E-Mail karger@karger.com & $\begin{array}{l}\text { This is an Open Access article licensed under the Creative Commons } \\
\text { Attribution-NonCommercial-4.0 International License (CC BY-NC) } \\
\text { (http://www.karger.com/Services/OpenAccessLicense), applicable to } \\
\text { the online version of the article only. Usage and distribution for com- } \\
\text { mercial purposes requires written permission. }\end{array}$
\end{tabular}

Results: Seventy percent of the patients had unilateral CCO. Iridocorneal adhesions (61 eyes, 52.1\%) and cataracts (22 eyes, $18.8 \%$ ) were the 2 most common ocular abnormalities. Systemic abnormalities were present in 5 patients (5.6\%), including growth retardation (4 patients) and congenital brain defects (1 patient). Eighty-five eyes (72.6\%) underwent penetrating keratoplasty (PK), and lamellar keratoplasty (LK) was performed in 30 (25.6\%) eyes. Forty-seven (95.9\%) eyes with Peters anomaly and all 16 eyes with sclerocornea received PK, and all 24 eyes with dermoids were treated with LK. Conclusion: Our study demonstrates that CCO has varied manifestations in infants and young children in China. A thorough medical history, careful clinical examination, and the use of accessory examinations such as ultrasound biomicroscopy are critical for the accurate diagnosis and classification of $\mathrm{CCO}$ and to provide guidance on therapeutic choices.

(c) 2019 The Author(s)

Published by S. Karger AG, Basel 


\section{Introduction}

Congenital corneal opacities (CCO) are a rare abnormality of the eye and caused by diverse factors [1-3]. In a clinicoepidemiological survey of congenital eye malformations in 1,124,654 consecutive births in Spain, Bermejo and Martínez-Frías [4] reported that CCO had a prevalence of 6 per 100,000 newborns. Epidemiological data for CCO are lacking in China; Shi et al. [5] studied 371 children (410 eyes) aged 14 years or younger who underwent corneal transplantation between 1994 and 2005 and found CCO in 53 eyes (12.9\%).

Due to its rarity, clinicians are not very familiar with the clinical features of CCO, which may cause delays and even omissions in diagnosis and treatment, resulting in severe visual impairment; this may even jeopardizing the overall development of infants and children $[2,3,6]$. Therefore, early detection, correct classification, and identification of other ocular and systemic abnormalities are important for appropriate therapy in order to minimize the risk of life-long visual impairment in patients with CCO [7]. In this retrospective single institution study, we investigated the clinicopathologic features and treatment characteristics of 90 CCO patients (117 eyes) who were 3 years and younger.

\section{Patients and Methods}

\section{Patients}

We retrospectively reviewed the clinical data of CCO patients who presented for the first time at the Corneal Disease Ward, Tongren Eye Center, Beijing, China, between January 1, 2017, and December 31, 2017. The patients from across China were referred to our center by primary and secondary care physicians for surgical treatment. All cases underwent a full clinical evaluation including anterior segment photography, high-frequency ultrasound biomicroscopy, and, if available, histopathology. Patients who presented with loss of transparency of the corneal tissue on anterior segment photography and had evident manifestations at the time of birth were diagnosed with CCO [1]. Patients who were no more than 3 years of age were included. Patients with acquired traumatic or nontraumatic corneal opacities were excluded.

The study protocol was approved by the Medical Ethics Committee of Beijing Tongren Hospital, and the study was conducted in accordance with the Declaration of Helsinki. No consent was required from the parents or legal guardians of the study subjects given the retrospective nature of the study. Patient data were anonymized in this report.

\section{Data Retrieval}

Data were collected from computerized medical records by entering the diagnosed disease as a key search word, and all the records were reviewed. Only data from the first visit were included

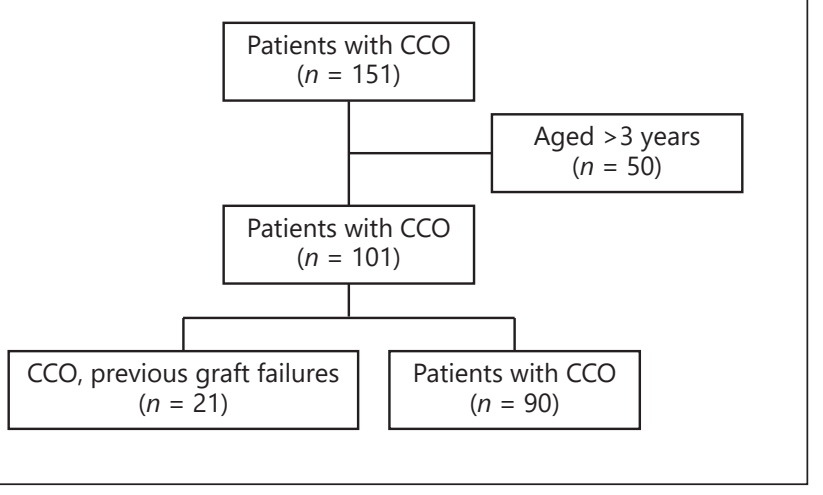

Fig. 1. The study flowchart. CCO, congenital corneal opacities.

to avoid the error of duplicated collection. Data on laterality, classification, associated ocular and systemic abnormalities, pathological changes, and treatment received were retrieved. Laterality (unilateral or bilateral) was diagnosed by clinically significant opacity observed by slit-lamp examination and, when possible, with the assistance of ultrasound biomicroscopy. Associated ocular abnormalities were diagnosed by slit-lamp examination and funduscopy, with the help of B-mode echography when the posterior segment was invisible due to CCO. The "STUMPED" method was used for the classification of CCO and confirmed by pathological examination [8]. Peters anomaly was further classified into 3 types $[9,10]$.

\section{Pathological Examination}

Surgical specimens were obtained from patients with CCO who underwent keratoplasty. Fresh opacity cornea tissues were placed in $10 \%$ neutral formalin immediately after excision. The tissues were dehydrated, paraffin-embedded, sectioned, routinely stained with hematoxylin and eosin, and examined under a light microscope.

\section{Surgery}

All the subjects included in this study underwent surgical treatment by a team of experienced corneal specialists at Beijing Tongren Eye Center. Consent for surgery was obtained from their parents or legal surrogates. Surgery methods included penetrating keratoplasty (PK), lamellar keratoplasty (LK), pupil reconstruction, and combined surgery.

\section{Statistical Analysis}

Data were expressed as number and percentage (\%) and analyzed using SPSS statistics 22.0 software (IBM, Armonk, NY, USA).

\section{Results}

\section{Patient Demographic and Demographic \\ Characteristics}

The study flowchart is shown in Figure 1. Totally, 151 CCO patients presented for the first time for treatment at our institution. Forty patients were excluded because they 
Fig. 2. Four images from a 4-month-old boy diagnosed with Peters anomaly. a Slit lamp diffuse light examination shows that the cornea is partially turbid and the pupil is not visible. $\mathbf{b}$ UBM examination shows visible punctate iris and corneal endothelial adhesion. c B-mode ultrasound examination shows no obvious intraocular abnormalities. d Intraoperative photos confirm the presence of iris and opacity corneal punctate anterior adhesion.

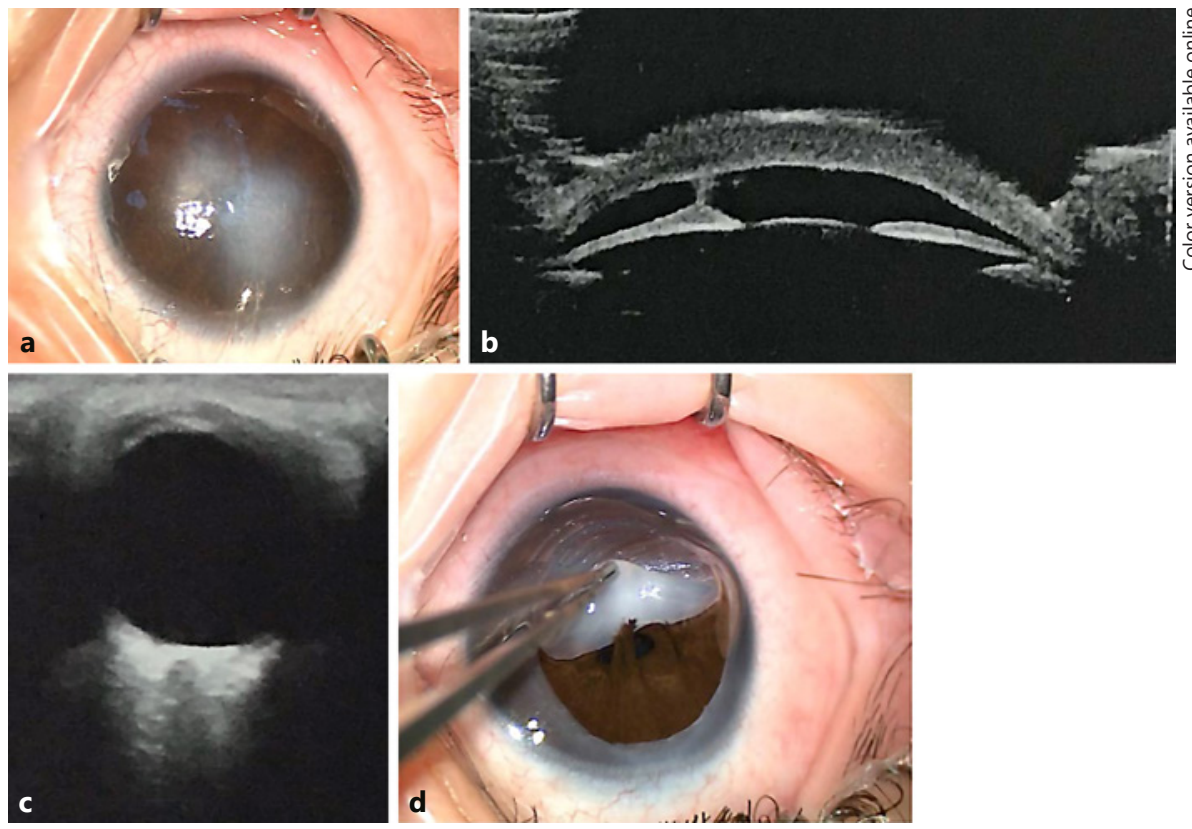

Table 1. STUMPED classification of CCO of the study population

\begin{tabular}{lccc}
\hline Classification & $\begin{array}{l}\text { All patients } \\
n(\%)\end{array}$ & $\begin{array}{l}\text { Unilateral } \\
\text { CCO, } n\end{array}$ & $\begin{array}{l}\text { Bilateral } \\
\text { CCO, } n\end{array}$ \\
\hline Peters' anomaly & $32(35.6)$ & 15 & 17 \\
Dermoids & $24(26.7)$ & 24 & - \\
Sclerocornea & $15(16.7)$ & 14 & 1 \\
Congenital glaucoma & $2(2.2)$ & 1 & 1 \\
Microphthalmia & $2(2.2)$ & - & 2 \\
Congenital anterior staphyloma & $1(1.1)$ & - & 1 \\
Axenfeld-Rieger syndrome & $1(1.1)$ & - & 1 \\
Congenital corneal endothelial dystrophy & $1(1.1)$ & - & 1 \\
Idiopathic & $12(13.3)$ & 9 & 3 \\
\hline Total & $\mathbf{9 0 ( 1 0 0 )}$ & $\mathbf{6 3}$ & $\mathbf{2 7}$ \\
\hline
\end{tabular}

CCO, congenital corneal opacities.

were aged above 3 years, 21 patients were excluded because their corneal opacities were acquired (the original disease was CCO but previous graft failure occurred). Finally, 90 patients (117 eyes) were included in the study. They included 50 (55.6\%) boys and 40 (44.4\%) girls with a mean age of $13.08 \pm 9.48$ months (range 3-36 months). The majority (70.0\%) of the patients had unilateral CCO while $30.0 \%$ had bilateral CCO. The STUMPED classifications of CCO in the study cohort are shown in Table 1. The top 3 CCOs were Peters anomaly (35.6\%), dermoids (26.7\%), and sclerocornea (16.7\%), accounting for $79.0 \%$ of all CCO cases. In unilateral CCOs, dermoids (38.1\%),
Peters anomaly (23.8\%), and sclerocornea (22.2\%) were the most common anomaly. In bilateral CCOs, Peters anomaly $(63.0 \%)$ was the most common CCO followed by idiopathic CCO (11.1\%) and microphthalmia (7.4\%; Fig. 2).

\section{Ocular and Systemic Abnormalities}

Iridocorneal adhesions (61 eyes, 52.1\%) and cataracts (22 eyes, $18.8 \%$ ) were the 2 most common ocular abnormalities (Table 2). Most of these abnormalities appeared in patients with Peters anomaly. Thirty patients (54 eyes) had type 1 Peters anomaly, 1 patient 

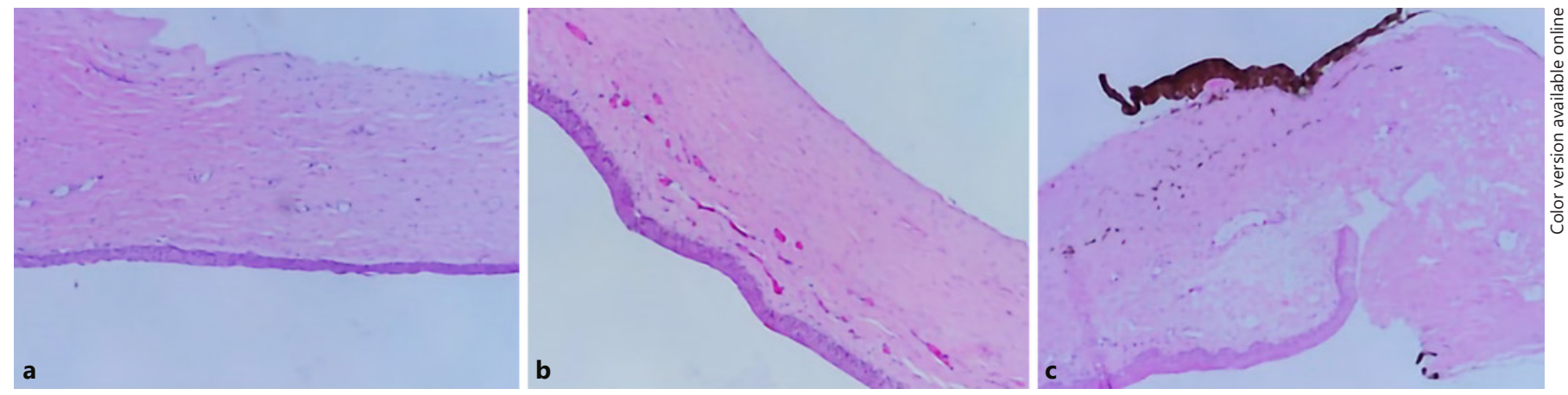

Fig. 3. Images of different pathological types. a Defects are shown in the Descemet's membrane and endothelium. b A large number of new blood vessels are visible in the stromal layer, and the tissue is loosely arranged irregularly. c Defects in the Descemet's membrane and endothelium and pigment particles are visible in the stroma and on the place where the endothelium defect is present.

Table 2. Ocular and systemic abnormalities in the study population, eyes (\%)

\begin{tabular}{|c|c|c|}
\hline & Total, $n(\%)$ & Classification ratio \\
\hline \multicolumn{3}{|l|}{ Ocular abnormalities } \\
\hline Iridocorneal adhesions & $61(52.1)$ & $71.1 \%$ seen in Peters anomaly \\
\hline Cataracts & $22(18.8)$ & Peters anomalies and idiopathic cataracts each account for $31 \%$ \\
\hline Iris neovascularization & $2(1.7)$ & Seen in Peters anomaly and microphthalmia \\
\hline Coloboma & $4(3.4)$ & $75 \%$ seen in idiopathic cases \\
\hline Lens dislocation & $3(2.6)$ & Seen in Peters anomaly, idiopathic cases, and A-R syndrome \\
\hline Papilledema & $2(1.7)$ & Seen in Peters anomaly \\
\hline Retina detachment & $1(0.9)$ & Seen in A-R syndrome \\
\hline PHPV & $3(2.6)$ & $33.3 \%$ seen in Peters anomaly \\
\hline \multicolumn{3}{|l|}{ Systemic abnormalities } \\
\hline Growth retardation & $4(3.4)$ & Seen in Peters-plus syndrome and idiopathic \\
\hline Congenital brain defects & $1(0.9)$ & Seen in Peters-plus syndrome \\
\hline
\end{tabular}

PHPV, persistent hyperplastic primary vitreous; classification ratio, proportion of classification in patients with abnormalities.

( 1 eye) had type 2 Peters anomaly, and 1 patient ( 2 eyes) had Peters-plus syndrome. Other ocular abnormalities included iris neovascularization ( 2 eyes), coloboma (4 eyes), lens dislocation ( 3 eyes), papilledema ( 2 eyes), retinal detachment (1 eye), and persistent hyperplastic primary vitreous ( 3 eyes). Systemic abnormalities were present in 5 patients $(5.6 \%)$, including growth retardation (4 patients) and congenital brain defects (1 patient). H\&E staining revealed 3 typical manifestations: defect or discontinuous of corneal endothelial and Descemet's membrane (59 eyes, 69.4\%), visible neovascular lumens in the corneal stroma (7 eyes, 8.2\%), and visible pigment granules in the corneal stroma (5 eyes, 5.9\%; Fig. 3).

Clinicopathologic Features of CCO in Children

\section{Treatment Characteristics}

The treatment characteristics of the study cohort are shown in Table 3. Eighty-five eyes (72.6\%) underwent $\mathrm{PK}$, including $\mathrm{PK}$ alone (59.0\%) and PK combined with extracapsular cataract extraction (ECCE; 13.7\%). LK including deep anterior LK was performed in 30 (25.6\%) eyes. Forty-seven (95.9\%) eyes with Peters anomaly and all 16 eyes with sclerocornea received PK alone or with ECCE. All 24 eyes with dermoids were treated with LK including deep anterior LK.

\section{Follow-Up}

The patients were followed at an interval of 3 or 4 months. At the time of writing this paper, pupil recon- 
Table 3. Treatment characteristics of CCO patients in the study per number of eyes

\begin{tabular}{|c|c|c|c|c|c|c|c|c|c|c|}
\hline Treatment & Total & $\begin{array}{l}\text { Peters } \\
\text { anomaly }\end{array}$ & Dermoids & Sclerocornea & $\begin{array}{l}\text { Congenital } \\
\text { glaucoma }\end{array}$ & Microphthalmia & $\begin{array}{l}\text { Congenital } \\
\text { corneal } \\
\text { staphyloma }\end{array}$ & $\begin{array}{l}\text { Axenfeld- } \\
\text { Rieger } \\
\text { syndrome }\end{array}$ & $\begin{array}{l}\text { Congenital } \\
\text { corneal } \\
\text { endothelial } \\
\text { dystrophy }\end{array}$ & $\begin{array}{l}\text { Idio- } \\
\text { pathic }\end{array}$ \\
\hline LK including DALK & $30(25.6)$ & - & 24 & - & - & - & - & - & - & 6 \\
\hline PK alone & $69(59.0)$ & 42 & - & 14 & 3 & 2 & 2 & - & 2 & 4 \\
\hline $\mathrm{PK}+\mathrm{ECCE}$ & $16(13.7)$ & 5 & - & 2 & - & 2 & - & 2 & - & 5 \\
\hline Total & $117(100)$ & 49 & 24 & 16 & 3 & 4 & 2 & 2 & 2 & 15 \\
\hline
\end{tabular}

LK, lamellar keratoplasty; DALK, deep anterior lamellar keratoplasty; PK, penetrating keratoplasty; ECCE, extracapsular cataract extraction; CCO, congenital corneal opacities.

struction and LK had a success rate of $100 \%$ and no serious postoperative complications were reported. Postoperative complications of $\mathrm{PK}$ included graft rejection $(22.4 \%, 19 / 85)$, glaucoma $(17.7 \%, 15 / 85)$, persistent corneal epithelial defect $(9.4 \%, 8 / 85)$, and retinal detachment $(1.2 \%, 1 / 85)$.

\section{Discussion}

The current study analyzed the clinicopathologic and treatment characteristics of 90 Chinese patients with (117 eyes) who were aged 3 years or younger and showed that the majority of CCO was unilateral and Peters anomaly, dermoids, and sclerocornea were the 3 most frequent CCOs, and iridocorneal adhesions and cataracts were the 2 most common abnormalities in the cohort.

Although the incidence of CCO is extremely low in newborns, visual impairment in these pediatric cases has lifelong implications, and therefore, it is important that CCO be detected early, diagnosed accurately and treated appropriately [1-3]. Thus, ophthalmologists must identify the classification of CCO according to medical history and clinical manifestations, and confirm ocular and systemic abnormalities through suitable examinations and provide appropriate treatment (medical or surgery) by considering multiple factors.

$\mathrm{CCO}$ was bilateral in 3 out of 10 patients in the present study, which is markedly lower than that $(73.6 \%)$ reported by Shigeyasu et al. [15] who only studied anterior segment CCO patients [11], thus excluding patients with dermoids that are predominantly unilateral and accounted for $26.7 \%$ of our population. In our series, Peters anomaly, dermoids, and sclerocornea occur in approxi- mately 8 out of 10 patients, which is similar to but higher than that (73.7\%) reported by Rezende et al. [3]. Our study included only surgical patients, and outpatients with sclerocornea who did not undergo surgery were excluded from the analysis. The importance of evaluation of unilateral and bilateral CCO cases lies in its prediction of postoperative recovery of vision. Mataftsi et al. [11] found that children with bilateral CCO had better recovery of vision than children with unilateral CCO post PK. We recommend that for cases of bilateral CCO, corneal transplant surgery be undertaken as early as possible, while for cases of unilateral $\mathrm{CCO}$, the decision to proceed with surgery be made after comprehensive evaluation of each case and educating the parents or legal guardians of the children.

Five (5.6\%) of our patients had systemic abnormalities, including growth retardation in 4 patients, which may be associated with aberrant gene expression or impact of impaired visual development that impedes cognitive development and development of the motor and nervous systems $[3,6]$. Ocular abnormalities were often seen in patients with Peters anomaly, which was the most frequent $\mathrm{CCO}$ in our cohort and was frequently associated with iridocorneal adhesions and cataracts, lending support to the theory that Peters anomaly is caused by abnormal development of the embryonic mesenchymal cell layer [12]. Discovery of ocular abnormalities may not only aid in making an accurate diagnosis but may also help physicians predict the prognosis of cases of CCO. Zaidman et al. [13] found that children with CCO combined with glaucoma had poor vision postoperatively.

The use of STUMPED for classifying CCO is not with its problems, which includes both signs (ulcer, sclerocornea, and Peters anomaly) and diagnoses (trauma, meta- 
bolic disorders, endothelial dystrophy, and dermoids) for classification of CCO. Such categorization may yield wrong clinical phenotypes when evaluated by high-frequency ultrasound [14]. In our series, we used ultrasound biomicroscopy to aid clinical analysis of causes of CCO. Furthermore, insight from ultrasound biomicroscopy could also provide guidance to surgery as surgeons can anticipate the presence of iridocorneal adhesions or anterior adhesions of the lenses and plan surgery accordingly [15].

Though PK, including PK combined with ECCE, was performed in the majority (72.6\%) of our patients, not all infants or younger children should undergo PK given inherent issues associated with the procedure and the unique anatomic structure of the eye in infants and younger children. Generally, we recommend surgery, if after pupil dilation with mydriatic drugs, the pupil is still occluded by corneal opacities and the pupil area cannot be penetrated. In other words-corneal transplant surgery is indicated when light cannot reach the retina and stimulate visual development with conservative treatment. Studies have shown that PK may not need to be performed as early as possible $[16,17]$. In our study, for those not indicated for PK, we chose pupil reconstruction as an alternative if the opacity area was limited, and the peripheral cornea was clear and cataract-free. Conservative treatment such as with atropine was given instead of surgery if the patient's condition permitted. All 24 eyes with dermoids in our series were treated with LK, which is similar to the study by Watts et al. [18].

After the corneal graft becomes stabilized after-corneal transplant surgery, the children under our care are transferred to the refractive specialist to undergo amblyopia training. Glasses are prescribed if the children are cooperative, especially in cataract combined cases with no lens inserted.
This work has some limitations. Our hospital is a leading tertiary eye care center in China, and most of our patients were referred to us for surgery by secondary and primary care physicians. Only a small proportion of the referred patients $(<10 \%)$ were treated conservatively. Therefore, our experience may not be applicable to secondary and primary care settings where the proportion of surgical patients is noticeably smaller. First of all, given the financial constraints of many patients and the limited availability of genetic study tools, we did not carry out gene sequencing studies on our patients. Such gene sequencing information assists in correct diagnosis of $\mathrm{CCO}$ [19]. Classification of CCO by STUMPED method is popular in China $[18,20]$, and phenotyping and genotyping using Nischal method are still not yet available [21]. However, to avoid diagnostic inaccuracies using STUMPED method, we used ultrasound biomicroscopy to aid clinical analysis of causes of CCO. In addition, considering the short duration of follow-up in the current study which was done at 3 or 4 month intervals, more data will be added for future analysis as we continue to follow-up the study cohort in the long term.

\section{Conclusion}

Our study demonstrates that CCO has varied manifestations in infants and younger children 3 years of age and under in China. Peters anomaly remains a predominant abnormality in this population of CCO patients, and is mostly managed by PK. A thorough medical history, careful clinical examination, and the use of accessory examinations such as ultrasound biomicroscopy are critical for accurate diagnosis and classification of CCO and to provide guidance for choice of therapeutic approaches.

\section{References}

1 Wilson FM. Congenital anomalies of the cornea and conjunctiva. In: Smolin G, Thoft RA, editors. The cornea. Boston: Little, Brown and Co; 1994. pp. 535-54.

2 Cotran PR, Bajart AM. Congenital corneal opacities. Int Ophthalmol Clin. 1992;32(1): 93-105.

3 Rezende RA, Uchoa UB, Uchoa R, Rapuano CJ, Laibson PR, Cohen EJ. Congenital corneal opacities in a cornea referral practice. Cornea. 2004 Aug;23(6):565-70.

4 Bermejo E, Martínez-Frías ML. Congenital eye malformations: clinical-epidemiological analysis of 1,124,654 consecutive births in Spain. Am J Med Genet. 1998 Feb;75(5):497-504.
5 Shi W, Jin H, Li S, Liu M, Xie L. Indications of paediatric keratoplasty in north China. Clin Exp Ophthalmol. 2007 Nov;35(8):724-7.

6 Zhang Y, Liu Y, Liang Q, Miao S, Lin Q, Zhang J, et al. Indications and outcomes of penetrating keratoplasty in infants and children of beijing, china. Cornea. 2018 Oct; 37(10):1243-8.

7 Dale NJ, Tadić V, Sonksen P. Social communicative variation in 1-3-year-olds with severe visual impairment. Child Care Health Dev. 2014 Mar;40(2):158-64.

8 Katzman LR, Reiser BJ. Pediatric corneal opacities. 2016
9 Sonksen PM, Dale N. Visual impairment in infancy: impact on neurodevelopmental and neurobiological processes. Dev Med Child Neurol. 2002 Nov;44(11):782-91.

10 Arffa RC. Congenital anomalies. In: Arffa RC, editor. Grayson's Diseases of the Cornea. St Louis: Mosby; 1997.

11 Mataftsi A, Islam L, Kelberman D, Sowden JC, Nischal KK. Chromosome abnormalities and the genetics of congenital corneal opacification. Mol Vis. 2011;17:1624-40.

12 Maillette de Buy Wenniger-Prick LJ, Hennekam RC. The Peters' plus syndrome: a review. Ann Genet. 2002 Apr-Jun;45(2):97103. 
13 Zaidman GW, Flanagan JK, Furey CC. Longterm visual prognosis in children after corneal transplant surgery for Peters anomaly type I. Am J Ophthalmol. 2007 Jul;144(1):104-8.

14 Nischal KK, Naor J, Jay V, MacKeen LD, Rootman DS. Clinicopathological correlation of congenital corneal opacification using ultrasound biomicroscopy. Br J Ophthalmol. 2002 Jan;86(1):62-9.

15 Shigeyasu C, Yamada M, Mizuno Y, Yokoi T, Nishina S, Azuma N. Clinical features of anterior segment dysgenesis associated with congenital corneal opacities. Cornea. 2012 Mar;31(3):293-8.
16 Ozeki H, Shirai S, Nozaki M, Sakurai E, Mizuno S, Ashikari M, et al. Ocular and systemic features of Peters' anomaly. Graefes Arch Clin Exp Ophthalmol. 2000 Oct;238(10):833-9.

17 Townsend WM. Congenital anomalies of the cornea. In: Kaufman HE, Baron BA, McDonald $\mathrm{MB}$, editors. The cornea. London: Butterworth-Heinemann; 1999.

18 Watts P, Michaeli-Cohen A, Abdolell M, Rootman D. Outcome of lamellar keratoplasty for limbal dermoids in children. J AAPOS. 2002 Aug;6(4):209-15.
19 Nischal KK. Genetics of congenital corneal opacification-impact on diagnosis and treatment. Cornea. 2015 Oct;34(suppl 10):S24-34.

20 Dana MR, Schaumberg DA, Moyes AL, Gomes JA. Corneal transplantation in children with Peters anomaly and mesenchymal dysgenesis. Multicenter Pediatric Keratoplasty Study. Ophthalmology. 1997 Oct;104(10): 1580-6.

21 Nischal KK. A new approach to the classification of neonatal corneal opacities. Curr Opin Ophthalmol. 2012 Sep;23(5):344-54. 